\title{
Stability of Schrödinger Eigenvalue Problems
}

\author{
E. Vock and W. Hunziker
}

Institut für Theoretische Physik, ETH Hönggerberg, CH-8093 Zürich, Switzerland

\begin{abstract}
We derive a general stability criterion for discrete eigenvalues of Schrödinger operators, such as $A(\kappa)=p^{2}+V(x, \kappa)$, using only strong continuity of $A(\kappa)$ and $A^{*}(\kappa)$ in the perturbation parameter $\kappa$. The theory is developed for non-selfadjoint operators and illustrated with examples like the anharmonic oscillator, the Stark and the Zeeman effect. The principal tools are Weyl's criterion for the essential spectrum and a construction due to Enss [5]. They are also used to extend the classical invariance theorems for the essential spectrum to certain singular perturbations, including some local perturbations of the Laplacian by differential operators of arbitrary high order.
\end{abstract}

\section{Introduction}

We introduce the stability problems treated in this paper with the following familiar examples:

Example 1 (Anharmonic oscillator [11]).

$$
A(\kappa)=p^{2}+x^{2}+\kappa x^{4}
$$

on $L^{2}\left(R^{1}\right)$, with complex $\kappa$ in the sector

$$
-\pi+\varepsilon \leqq \arg \kappa \leqq \pi-\varepsilon \quad(\varepsilon>0) .
$$

Example 2 (Diatomic molecular ion [2]).

$$
A(\kappa)=p^{2}-|x|^{-1}-|x-\kappa|^{-1}
$$

on $L^{2}\left(R^{3}\right)$, with $\kappa \in R^{3}$.

Example 3 (Stark effect [6]).

$$
A(\kappa)=e^{-2 \theta} p^{2}-e^{-\theta}|x|^{-1}+\kappa e^{\theta} x_{1}
$$


on $L^{2}\left(R^{3}\right)$, where $\theta$ is an auxiliary complex dilation parameter and $\kappa$ complex in the sector

$$
0<\varepsilon \leqq 3 \operatorname{Im} \theta+\arg \kappa \leqq \pi-\varepsilon .
$$

Example 4 (Zeeman effect [3]).

$$
A(\kappa)=(p-\kappa \wedge x)^{2}-|x|^{-1}
$$

on $L^{2}\left(R^{3}\right)$, with $\kappa \in R^{3}$.

Common to all these examples is the failure of analytic perturbation theory at $\kappa_{0}=0$. For (1.1) and (1.3) it is known that the resolvent $R(z, \kappa)=(z-A(\kappa))^{-1}$ is norm-continuous :

$$
\lim _{\kappa \rightarrow \kappa_{0}}\left\|R\left(z_{0}, \kappa\right)-R\left(z_{0}, \kappa_{0}\right)\right\|=0
$$

for some $z_{0}$ in the resolvent set $\varrho\left(A\left(\kappa_{0}\right)\right)$. By the resolvent identity

$$
R\left(z_{0}, \kappa\right)-R(z, \kappa)=\left(z-z_{0}\right) R\left(z_{0}, \kappa\right) R(z, \kappa) .
$$

(1.7) extends to all $z \in \varrho\left(A\left(\kappa_{0}\right)\right)$ [8], so that any discrete eigenvalue of $A\left(\kappa_{0}\right)$ (i.e. any isolated eigenvalue of finite multiplicity) is stable in the following sense:

Definition [8]. A discrete eigenvalue $\lambda$ of $A\left(\kappa_{0}\right)$ is stable with respect to the family $A(\kappa)$ if

(i) given any sufficiently small $r>0$,

$$
\Gamma_{r}=\{z|| z-\lambda \mid=r\} \subset \varrho(A(\kappa))
$$

for all $\kappa$ in some neighbourhood of $\kappa_{0}$, and

$$
\lim _{\kappa \rightarrow \kappa_{0}}\left\|P(\kappa)-P\left(\kappa_{0}\right)\right\|=0
$$

where

$$
P(\kappa)=(2 \pi i)^{-1} \oint_{I_{r}} d z R(z, \kappa)
$$

is the spectral projection of $A(\kappa)$ corresponding to the part of the spectrum enclosed in $\Gamma_{r}$.

Since (ii) implies $\operatorname{dim} P(\kappa)=\operatorname{dim} P\left(\kappa_{0}\right)$ for $\kappa$ close to $\kappa_{0}$, a stable eigenvalue is the limit of a group of perturbed eigenvalues with the same total algebraic multiplicity [8]. Discrete eigenvalues of $A\left(\kappa_{0}\right)$ may still be stable when (1.7) fails : this situation is met in Examples 3 and 4, where the resolvent is strongly (but not norm-) continuous at $\kappa_{0}=0$ :

$$
\operatorname{S-lim}_{\kappa \rightarrow \kappa_{0}} R(z, \kappa)=R\left(z, \kappa_{0}\right)
$$

for all $z$ in some open set $\Delta C \varrho\left(A\left(\kappa_{0}\right)\right)$. In general this is not sufficient for stability for the following reasons :

First, (1.11) does not extend automatically to all $z \in \varrho\left(A\left(\kappa_{0}\right)\right)$, i.e. a given discrete eigenvalue of $A\left(\kappa_{0}\right)$ need not be embedded in $\Delta$. Secondly, if $\Gamma_{r}$ is a circle contained in $\Delta$, it follows from (1.10) that

$$
\underset{\kappa \rightarrow \kappa_{0}}{S-\lim _{\kappa}} P(\kappa)=P\left(\kappa_{0}\right) .
$$


This only implies that $\operatorname{dim} P(\kappa) \geqq \operatorname{dim} P\left(\kappa_{0}\right)$ for $\kappa$ close to $\kappa_{0}$, so that $A(\kappa)$ may have more eigenvalues than $A\left(\kappa_{0}\right)$ in the circle $\Gamma_{r}$. An example is

$$
A(\kappa)=p^{2}+V(x-\kappa)
$$

on $L^{2}\left(R^{1}\right)$ with $\kappa \in R^{1}$. Let $V$ be a real $C_{0}$-function such that $A(0)$ has a simple eigenvalue $\lambda<0$ with eigenfunction $u(x),\|u\|=1$. Since $A(\kappa)$ is unitarily equivalent to $A(0)$ by a translation, it follows that $\|R(z, \kappa)\|=\|R(z, 0)\|$ for all $z \in \varrho(A(0))$. As we will see below (Lemma 1.2), this implies

$$
\lim _{\kappa \rightarrow \infty} R(z, \kappa)=\left(z-p^{2}\right)^{-1}
$$

for all $z \in \varrho(A(0))$. Therefore, if $\Gamma_{r}$ is a sufficiently small circle around $\lambda,(1.12)$ reads

$$
S \text { - } \lim _{\kappa \rightarrow \infty} P(\kappa)=0,
$$

since $A(\infty)=p^{2}$ has spectrum $[0, \infty)$. This is also evident from the explicit form

$$
P(\kappa): \psi \rightarrow(u(\kappa), \psi) u(\kappa),
$$

where $u(x, \kappa)=u(x-\kappa)$ is the translated eigenfunction.

In Examples 3 and 4 it is possible, however, to derive norm-continuity of the relevant part of the resolvent from (1.11) and a compactness argument [3,6]. Briefly, let

$$
A(\kappa)=A_{0}(\kappa)+V,
$$

where $V$ is the term involving the Coulomb potential $|x|^{-1}$ in (1.4) or (1.6). $R_{0}(z, \kappa)$ $=\left(z-A_{0}(\kappa)\right)^{-1}$ is strongly (not norm-) continuous at $\kappa_{0}=0$ for all $z$ in some open set $\Delta_{0}$. However, $V R_{0}(z, \kappa)$ is compact and norm-continuous at $\kappa_{0}$. Applying the Fredholm theory to the equation

$$
R(z, \kappa)=R_{0}(z, \kappa)+R(z, \kappa) V R_{0}(z, \kappa),
$$

it then follows that $R(z, \kappa)-R_{0}(z, \kappa)$ is norm-continuous as $\kappa \rightarrow \kappa_{0}$ whenever $z \in \Delta_{0}$ is not an eigenvalue of $A\left(\kappa_{0}\right)$. This proves stability for any discrete eigenvalue $\lambda \in \Delta_{0}$ of $A\left(\kappa_{0}\right)$, since the subtraction $R_{0}(z, \kappa)$ does not contribute to $P(\kappa)$ in $(1.10)$. The argument can be extended to $N$-particle systems by using a "connected" resolvent equation instead of $(1.14)[4,7]$.

To complete our summary of existing stability proofs for Schrödinger eigenvalue problems, we should mention variational methods. Although restricted to selfadjoint families, they have the great advantage to work with $A(\kappa)$ directly rather than with the resolvent, so that strong continuity of $A(\kappa)$ on a suitable domain suffices to prove stability. The limit $\kappa \rightarrow \infty$ in Example 2 (and more general "cluster" limits of molecular spectra) have been treated in this way [9].

In this paper we develop a general stability theory for discrete eigenvalues of Schrödinger-type operators, which allows us to treat all these examples from a common point of view. It is not restricted to selfadjoint families, but like the variational method it works with $A(\kappa)$ directly and requires only strong continuity in the following sense: 
Hypothesis 1. Let $G$ be a first countable topological space and $\kappa \rightarrow A(\kappa)$ a function on $G$ taking values in the closed operators on a Hilbert space $\mathscr{H}$. For a given $\kappa_{0} \in G$ we assume:

$$
\lim _{\kappa \rightarrow \kappa_{0}} A(\kappa) u=A\left(\kappa_{0}\right) u
$$

for all $u$ in some core $D$ of $A\left(\kappa_{0}\right)$, and

$$
\lim _{\kappa \rightarrow \kappa_{0}} A^{*}(\kappa) u=A^{*}\left(\kappa_{0}\right) u
$$

for all $u$ in some core $D^{*}$ of $A^{*}\left(\kappa_{0}\right)$.

Implicit are the domain conditions $D \subset D(A(\kappa))$ and $D^{*} C D\left(A^{*}(\kappa)\right)$ for $\kappa$ close to $\kappa_{0}$. In the examples given above it is known that $C_{0}^{\infty}\left(R^{v}\right)$ is a core of $A(\kappa)$ and $A^{*}(\kappa)$, so that Hypothesis 1 is satisfied. This alone is of course not sufficient for stability, but it is the only continuity-condition we will use. The nature of the other conditions is illustrated by the following class of results:

Example 5 (Schrödinger operators with core $C_{0}^{\infty}$ ). We consider the formal operator

$$
A(V)=p^{2}+V(x)
$$

on $L^{2}\left(R^{v}\right)$, where the potential $V$ plays the role of the perturbation parameter. $V$ is restricted to a subset $G$ of $L_{\text {loc }}^{2}\left(R^{\nu}\right)$, which is determined by positive numbers $\xi, \eta, \varepsilon$ via the following conditions:

(i) Let $A_{0}(V)$ be the operator (1.17) restricted to the domain $C_{0}^{\infty}\left(R^{v}\right)$. Then

$$
\begin{aligned}
\left(u, p^{2} u\right) \leqq & \xi\left\{\cos \gamma(V) \operatorname{Re}\left(u, A_{0}(V) u\right)\right. \\
& \left.+\sin \gamma(V) \operatorname{Im}\left(u, A_{0}(V) u\right)+\eta(u, u)\right\}
\end{aligned}
$$

for some $\gamma(V)$ in the range $|\gamma| \leqq \pi / 2-\varepsilon$ and all $u \in C_{0}^{\infty}\left(R^{v}\right)$. In particular, $A_{0}(V)$ has numerical range in some halfplane contained in

$$
Q=\{z \in C \mid-\pi+\varepsilon \leqq \arg (z+\eta / \sin \varepsilon) \leqq \pi-\varepsilon\} .
$$

(ii) $\operatorname{Ran}\left(z-A_{0}(V)\right)$ is dense for some $z \notin Q$. Therefore $A_{0}(V)$ has a closure $A(V)$ with spectrum in some halfplane contained in $Q$.

The topology on $G$ is the $L_{\text {loc }}^{2}$-topology, i.e. the functions $V \rightarrow V \psi$ are continuous from $G$ to $L^{2}\left(R^{v}\right)$ for any $\psi \in C_{0}^{\infty}\left(R^{v}\right)$. Hypothesis 1 is then satisfied with $D=D^{*}=C_{0}^{\infty}\left(R^{v}\right)$.

Theorem 1.1. Let $A(V)$ be the family of operators given in Example 5, and

$$
E_{n}(V)=\left\{(u, A(V) u) \mid u \in C_{0}^{\infty}\left(R^{v}\right),\|u\|=1, u(x)=0 \quad \text { for } \quad|x|<n\right\} .
$$

Let $\Omega$ be an open complex set and $G_{\Omega}$ a subspace of potentials $V \in G$ such that

$$
\Omega \cap E_{n}(V)=\emptyset
$$

for some fixed (arbitrarily large) $n$. Then for each $V_{0} \in G_{\Omega}$, the spectrum of $A\left(V_{0}\right)$ in $\Omega$ is purely discrete and each eigenvalue $\lambda \in \Omega$ of $A\left(V_{0}\right)$ is stable with respect to the family $\left\{A(V) \mid V \in G_{\Omega}\right\}$.

Remarks. In the language of quantum mechanics: $A(V)$ has purely discrete spectrum in any complex energy region $\Omega$ where tunneling to infinity is forbidden 
by energy conservation. As long as this barrier exists, the eigenvalues in $\Omega$ are stable when $V$ changes continuously in a $L_{\text {loc }}^{2}$ sense.

Explicit sufficient conditions for $V \in G$ are given in Sect. 6: Examples 1-3 are subfamilies of the form $A(\kappa)=A(V(\kappa))$ - except for a trivial numerical factor in (1.4). To apply Theorem 1.1 to these cases it therefore suffices to check the numerical range $E_{n}(\kappa)$ of $A(\kappa)$ for states $u \in C_{0}^{\infty}\left(R^{v}\right)$ with support in $\{|x|>n\}$ :

Example 1. $E_{n}(\kappa)$ is contained in the shaded region of Fig. 1:

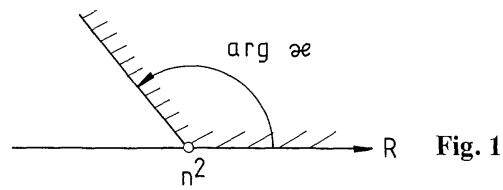

so that (1.20) is satisfied for any bounded set $\Omega$ if $n$ is sufficiently large. Therefore $A\left(\kappa_{0}\right)$ has purely discrete spectrum for any $\kappa_{0}$ in the sector (1.2), and all eigenvalues of $A\left(\kappa_{0}\right)$ are stable with respect to the family $A(\kappa)$.

The same arguments apply to more general cases like

$$
A(\kappa)=p^{2}+V_{1}(x)+\kappa V_{2}(x)
$$

on $L^{2}\left(R^{v}\right)$ with real $V_{1,2} \in L_{\text {loc }}^{2}\left(R^{v}\right)$ which are bounded below and grow to $+\infty$ as $|x| \rightarrow \infty$. Analytic perturbation theory works for $\kappa_{0}$ in the interior of the sector (1.2) but not at $\kappa_{0}=0$. Whether or not asymptotic perturbation theory applies in this case depends on the growth rate of $V_{2}(x)$.

Example 2. For given $\kappa_{0} \in R^{3}$, we restrict $\kappa$ to some finite ball around $\kappa_{0}$. Then (1.20) holds for some $n$ if $\Omega$ has distance $>0$ from the positive real axis. Hence $A\left(\kappa_{0}\right)$ has purely discrete spectrum in the complement of $[0, \infty)$ and each eigenvalue in this region is stable with respect to $A(\kappa)$. The same argument works for

$$
A(\kappa)=p^{2}+\sum_{k=1}^{N} V_{k}\left(x-\kappa_{k}\right)
$$

on $L^{2}\left(R^{3}\right), \kappa=\left(\kappa_{1} \ldots \kappa_{N}\right) \in R^{3 N}$, for complex $V_{k} \in L_{\text {loc }}^{2}\left(R^{3}\right)$ if $V_{k}(x) \rightarrow 0$ as $|x| \rightarrow \infty$. We note, however, that the "cluster" limits $\kappa \rightarrow \infty$ (in certain directions of $R^{3 N}$ ) cannot be obtained in this way.

Example 3. Theorem 1.1 applies to $A(\kappa, \theta) \exp (2 \theta)$ for $(\kappa, \theta)$ satisfying (1.5). For states $u$ with support in $\{|x|>n\},(u, A(\kappa, \theta) u)$ has distance $<\left|e^{-\theta} n^{-1}\right|$ from the shaded halfplane $S$ in Fig. 2:

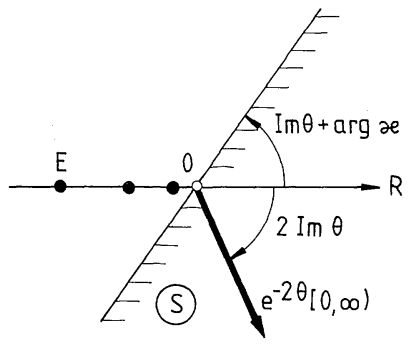

Fig. 2 
Therefore $A(\kappa, \theta)$ has purely discrete spectrum in the complement of $S$, and these eigenvalues are stable with respect to variations of $(\kappa, \theta)$. (In fact, it is known that $A(\kappa, \theta)$ has discrete spectrum in the whole plane if $\kappa \neq 0$, but the eigenvalues in $S$ are possibly unstable [6].)

Herbst's description of the Stark effect is as follows [6]: For real $\kappa \neq 0$ the Stark Hamiltonian $A(\kappa, 0)$ has spectrum $(-\infty,+\infty)$ without eigenvalues. The Stark effect becomes visible in the spectrum after a complex dilation $x \rightarrow e^{\theta} x$, which transforms $A(\kappa, 0)$ into $A(\kappa, \theta)$. For fixed $\kappa, A(\kappa, \theta)$ is analytic in $\theta$ in the region (1.5), with discrete eigenvalues independent of $\theta$ by the standard argument of dilation analyticity [1]. For $\kappa=0$ and $-\pi / 2<\operatorname{Im} \theta<\pi / 2$ the spectrum is shown in Fig. 2: its discrete part is the same as for $\theta=0$ (hydrogen eigenvalues), while the continuum $\exp (-2 \theta)[0, \infty)$ is rotated by an angle $-2 \operatorname{Im} \theta$. Each eigenvalue $E$ is stable with respect to $A(\kappa, \theta)$ as long as $E \notin S$, i.e. for

$$
0<\varepsilon \leqq \operatorname{Im} \theta+\arg \kappa \leqq \pi-\varepsilon
$$

intersected with (1.5). Taking the union of these regions in the $\kappa$-plane over $|\operatorname{Im} \theta|$ $<\pi / 2$, we find that the perturbed eigenvalues are defined in a sector $-\pi / 2+\varepsilon$ $\leqq \arg \kappa \leqq 3 \pi / 2-\varepsilon,|\kappa|<\delta$, where they converge to $E$ as $\kappa \rightarrow 0$. For real $\kappa$ these are the Stark resonances corresponding to $E$. We must refer to $[6,7]$ for further details.

After these introductory examples we give an outline of the problems which will occur in our discussion of stability. First, we remark that Hypothesis 1 implies strong continuity of the resolvent. Let

$$
\Delta=\left\{z \in C \mid R(z, \kappa) \text { exists and is uniformly bounded for } \kappa \text { close to } \kappa_{0}\right\} .
$$

We note that $\Delta$ is open. Given any compact $\Gamma \subset \Delta$ there exists a neighbourhood $U$ of $\kappa_{0}$ such that $R(z, \kappa)$ exists and is uniformly bounded for $(z, \kappa) \in \Gamma \times U$. This follows from (1.8) and a covering argument.

Lemma 1.2. Suppose that $A(\kappa)$ satisfies Hypothesis 1. Then

$$
\lim _{\kappa \rightarrow \kappa_{0}} R(z, \kappa) u=R\left(z, \kappa_{0}\right) u
$$

and

$$
\lim _{\kappa \rightarrow \kappa_{0}} R^{*}(z, \kappa) u=R^{*}\left(z, \kappa_{0}\right) u
$$

for any $u \in \mathscr{H}$ if and only if $z \in \Delta$. In both cases the convergence is locally uniform in $z \in \Delta$.

Proof. Fix $z \in \Delta$. Since $R(z, \kappa)$ is uniformly bounded for $\kappa$ close to $\kappa_{0}$ it suffices to prove (1.22) for the dense set of vectors $u=\left(z-A\left(\kappa_{0}\right)\right) v, v \in D$. Then by Hypothesis 1 ,

$$
\left\|R(z, \kappa) u-R\left(z, \kappa_{0}\right) u\right\|=\left\|R(z, \kappa)\left(A(\kappa)-A\left(\kappa_{0}\right)\right) v\right\| \rightarrow 0
$$

as $\kappa \rightarrow \kappa_{0}$. Local uniformity in $z \in \Delta$ follows from (1.8). The proof of (1.23) is analogous. 
In proving stability of a given discrete eigenvalue $\lambda$ of $A\left(\kappa_{0}\right)$ we will proceed in three steps:

(i) First, we need to know that for $\kappa$ close to $\kappa_{0}$,

$$
\operatorname{dist}\left\{\lambda, \sigma_{\text {ess }}(A(\kappa))\right\} \geqq \varepsilon>0 \text {. }
$$

(ii) Next, we want to show that for some $\varepsilon>0$,

$$
\{z|0<| z-\lambda \mid<\varepsilon\} \subset \Delta .
$$

It then follows from Lemma 1.2 that

$$
S-\lim _{\kappa \rightarrow \kappa_{0}} P(\kappa)=P\left(\kappa_{0}\right),
$$

and

$$
\lim _{\kappa \rightarrow \kappa_{0}} P^{*}(\kappa)=P^{*}\left(\kappa_{0}\right) .
$$

(iii) Finally, we will have to prove

$$
\operatorname{dim} P(\kappa) \leqq \operatorname{dim} P\left(\kappa_{0}\right)
$$

for $\kappa$ close to $\kappa_{0}$, since this together with (1.26) implies (1.9) by a result of Kato [8, Chap VIII].

(i) calls for an effective method to control the essential spectrum. We will use Weyl's criterion (Sect. 2) and a construction of Enss [5], extended to nonselfadjoint operators (Sect. 3). This is applied in Sect. 4 to prove invariance properties of the essential spectrum under certain additive perturbations, including some local perturbations of the Laplacian by differential operators of arbitrary high order. After this digression we return to the stability of discrete eigenvalues in Sect. 5, showing that the problems (ii) and (iii) are in fact closely related to (i) and can be handled by similar methods. This leads to some general stability criteria (Theorems 5.4 and 5.8) which are our main results. Examples play a dominant role throughout this work, since our general framework (Hypothesis 2 and 3 ) is abstracted from concrete problems.

\section{Weyl's Criterion}

Let $A$ be a closed operator on a Hilbert space $\mathscr{H}$. We define its essential spectrum by

$$
\sigma_{\text {ess }}(A)=\sigma(A) \backslash \sigma_{p}(A),
$$

where $\sigma(A)$ is the spectrum and $\sigma_{p}(A)$ the set of all isolated eigenvalues of finite multiplicity (discrete spectrum). Let

$$
\begin{aligned}
& W(A)=\left\{\lambda \in C \mid\left\|(\lambda-A) u_{n}\right\| \rightarrow 0 \quad\right. \text { for some sequence } \\
&\left.u_{n} \in D(A),\left\|u_{n}\right\| \nrightarrow 0, u_{n} \rightarrow \vec{w} \text { (weak convergence) }\right\} .
\end{aligned}
$$

Following Weyl [13], $\left\{u_{n}\right\}$ is called a "characteristic sequence of $\lambda-A$ ". Sometimes it is convenient to normalize $\left\|u_{n}\right\|=1$. 
Theorem 2.1 (Weyl's criterion). Let $A$ be a closed operator on a Hilbert space $\mathscr{H}$. Then

(i) $W(A)$ is closed,

(ii) $W(A) \subset \sigma_{\text {ess }}(A)$,

(iii) boundary of $\sigma_{\mathrm{ess}}(A) \subset W(A)$.

Proof. First, let $A$ be a closed operator on a Banach space $X$. We quote from [8, Chap. IV, Sect. 5]:

Definition (Sect. 5, Subsect. 6).

$$
\Sigma_{e}=\left\{\lambda \in C \mid \operatorname{nul}^{\prime}(\lambda-A)=\operatorname{def}^{\prime}(\lambda-A)=\infty\right\} .
$$

This is Kato's definition of the essential spectrum, which differs from (2.1).

Theorem 5.9. nul' $^{\prime}(\lambda-A)$ is the greatest number $m \leqq \infty$ with the following property: for any $\varepsilon>0$ there exists an m-dimensional closed subspace $N_{\varepsilon} \subset D(A)$ such that $\|(\lambda-A) u\| \leqq \varepsilon\|u\|$ for all $u \in N_{\varepsilon}$.

Theorem 5.11. $\operatorname{nul}^{\prime}(\lambda-A)=\infty$ if and only if there exists a sequence $u_{n} \in D(A)$ with $\left\|u_{n}\right\|=1$ and $\left\|(\lambda-A) u_{n}\right\| \rightarrow 0$ which contains no convergent subsequence.

Problem 5.37. If $\lambda$ is a boundary point of $\varrho(A)$ then $\lambda \in \Sigma_{e}(A)$ unless $\lambda$ is an isolated point of $\sigma(A)$.

Theorem 5.28. If $\lambda$ is an isolated point of $\sigma(A)$ and $P$ the corresponding spectral projection then $\operatorname{dim} P<\infty$ if and only if $\operatorname{nul}^{\prime}(\lambda-A)<\infty$.

With these results we first show that Theorem 2.1 holds if we redefine

$$
W(A)=\left\{\lambda \in C \mid \operatorname{nul}^{\prime}(\lambda-A)=\infty\right\} .
$$

Proof. (i) follows from Theorem 5.9. (ii) Let $\lambda \in W(A)$. Then $\lambda \in \sigma(A)$ is evident and $\lambda \notin \sigma_{p}(A)$ follows from Theorem 5.28. (iii) Let $\lambda$ be a boundary point of $\sigma_{\text {ess }}(A)$. Then $\lambda$ is a boundary point of $\varrho(A)$ and $\lambda \notin \sigma_{p}(A)$. It then follows from Problem 5.37 and Theorem 5.28 that $\operatorname{nul}^{\prime}(\lambda-A)=\infty$.

It remains to show that the definitions (2.2) and (2.3) coincide if $X$ is a Hilbert space. More generally we have:

Lemma 2.2. Let $X$ be the dual of a Banach space $Y$ and $A$ a closed operator on $X$. Then $\operatorname{nul}^{\prime}(\lambda-A)=\infty$ if and only if there exists a sequence $v_{n} \in D(A)$ such that $\left\|v_{n}\right\| \nrightarrow 0$ and $v_{n}(y) \rightarrow 0$ for all $y \in Y$.

Proof. If such a sequence exists, it contains no convergent subsequence, hence $\operatorname{nul}^{\prime}(\lambda-A)=\infty$ by Theorem 5.11. Conversely, if $\operatorname{nul}^{\prime}(\lambda-A)=\infty$, let $u_{n}$ be a sequence as described in Theorem 5.11. Since the unit ball in $X$ is weak*-compact, we may assume (by passing to a subsequence) that $u_{n}(y) \rightarrow u(y)$ for some $u \in X$ and all $y \in Y$. Since $\left\{u_{n}\right\}$ contains no norm-convergent subsequence, there exists $\varepsilon>0$ such that $\left\|u_{n}-u_{m(n)}\right\|>\varepsilon$ for each $n$ and some $m(n)>n$. Therefore $v_{n}=u_{n}-u_{m(n)}$ has the properties claimed in Lemma 2.2. This concludes the proof of Theorem 2.1. 
Remarks. The proof shows that Theorem 2.1 holds in reflexive Banach spaces. We will use it mostly in the form of

Corollary 2.3. Let $\Omega$ be an open, connected complex set with $\Omega \cap W(A)=\emptyset$ and $\Omega \cap \varrho(A) \neq \emptyset$. Then $\Omega \cap \sigma_{\text {ess }}(A)=\emptyset$, i.e. $A$ has purely discrete spectrum in $\Omega$.

Proof. If $\Omega \cap \sigma_{\text {ess }}(A) \neq \emptyset$, then $\Omega$ contains a boundary point of $\sigma_{\text {ess }}(A)$. By Theorem 2.1(iii) this is in contradiction to the hypothesis $\Omega \cap W(A)=\emptyset$.

We remark in particular that $\sigma_{\text {ess }}(A)=W(A)$ if and only if each connected component of $C \backslash W(A)$ contains a point of $\varrho(A)$. The classical example is $A=A^{*}$ [13].

Finally we mention a spectral mapping property which will be used in Sect. 4 :

Lemma 2.4. Let $A$ be a closed operator on a Hilbert space $\mathscr{H}$ with $\varrho(A) \neq \emptyset$. Then the following are equivalent:

(i) $\lambda \in W(A)$,

(ii) $0 \neq(z-\lambda)^{-1} \in W\left((z-A)^{-1}\right)$ for some (and therefore all) $z \in \varrho(A)$.

Also equivalent are:

(iii) $A$ is unbounded,

(iv) $0 \in W\left((z-A)^{-1}\right)$ for some (and therefore all) $z \in \varrho(A)$.

Proof. Any characteristic sequence of $\lambda-A$ is a characteristic sequence of $(z-\lambda)^{-1}$ $-(z-A)^{-1}$ if $z \in \varrho(A)$. Conversely, if $\left\{v_{n}\right\}$ is a characteristic sequence of $\mu-(z-A)^{-1}, \mu \neq 0$, then $u_{n}=(z-A)^{-1} v_{n}$ is a characteristic sequence of $z-\mu^{-1}-A$. This proves (i) $\leftrightarrow$ (ii). (iv) $\rightarrow$ (iii) is evident. (iii) $\rightarrow$ (iv): Since $A$ is unbounded, $\left\|A u_{n}\right\| \rightarrow \infty$ for some sequence $u_{n} \in D(A)$ with $\left\|u_{n}\right\|=1$. Setting $v_{n}=(z-A) u_{n}\left\|(z-A) u_{n}\right\|^{-1}$ for some $z \in \varrho(A)$, we see that $\left\|(z-A)^{-1} v_{n}\right\| \rightarrow 0$ and

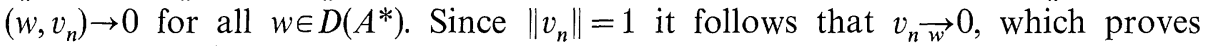
$0 \in W\left((z-A)^{-1}\right)$.

\section{The Construction of Enss}

The fact that characteristic sequences are non-unique leaves room for con-

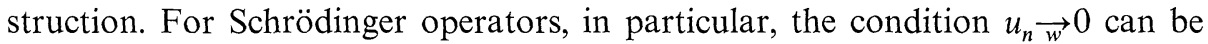
replaced by stronger support conditions such as $\operatorname{supp} u_{n} \subset\{|x|>n\}$ [5]. This is important since it shows that $W(A)$ depends only on the behavior of the local operator $A$ as $|x| \rightarrow \infty$. The following is an abstract and slightly generalized version of Enss' construction:

Hypothesis 2. Let $A$ be a closed operator on $\mathscr{H}$ with $\varrho(A) \neq \emptyset$ and resolvent $R(z)=(z-A)^{-1}$. We assume that $\left\{M_{n}\right\}$ is a sequence of equibounded operators on $\mathscr{H}$ with the following properties:

(i) If $\left\{u_{m}\right\}$ is a characteristic sequence of $\lambda-A$ then there exists $a>0$ such that

$$
\limsup _{m}\left\|M_{n} u_{m}\right\|>a \text { for all } n \text {. }
$$

(ii) $M_{n} D(A) \subset D(A)$ and

$$
\left[M_{n}, A\right] u=B_{n} u+K_{n} u
$$


for all $u \in D(A)$, where $K_{n}$ is compact and $\left\|B_{n} R(z)\right\| \rightarrow 0$ as $n \rightarrow \infty$ for some (and therefore all) $z \in \varrho(A)$.

Lemma 3.1. Suppose that $A$ satisfies Hypothesis 2. Then, if $\left\{u_{n}\right\}$ is a characteristic sequence of $\lambda-A$, so is $v_{n}=M_{n} u_{m(n)}$ provided that $m(n)$ is chosen sufficiently large for each $n$.

Proof. We normalize $\left\|u_{n}\right\|=1$ and let $z \in \varrho(A)$. Since $(z-A) u_{m} \underset{w}{\longrightarrow} 0$ it follows from

$$
\begin{aligned}
\left\|(\lambda-A) M_{n} u_{m}\right\| \leqq & \left\|M_{n}\right\|\left\|(\lambda-A) u_{m}\right\|+\left\|B_{n} R(z)(z-A) u_{m}\right\| \\
& +\left\|K_{n} R(z)(z-A) u_{m}\right\|
\end{aligned}
$$

and from Hypothesis 2 that we can choose $m(n)$ so large that

(i) $\left\|(\lambda-A) M_{n} u_{m}\right\|<|\lambda-z|\left\|B_{n} R(z)\right\|+n^{-1}$,

(ii) $\left\|M_{n} u_{m}\right\|>a / 2$, and

(iii) $\left|\left(e_{i}, M_{n} u_{m}\right)\right|<n^{-1}$ for $i=1 \ldots n$,

where $\left\{e_{i}\right\}$ is some fixed basis in the (separable) space $N$ spanned by the vectors $M_{p} u_{q}(p, q=1,2,3 \ldots)$. Since the sequence $v_{n}=M_{n} u_{m(n)}$ is bounded, it follows from (iii) that $\left(f, v_{n}\right) \rightarrow 0$ for all $f \in N$, while $\left(f, v_{n}\right)=0$ for $f \perp N$. Therefore $v_{n} \rightarrow 0$, which together with (i) and (ii) proves that $v_{n}$ is a characteristic sequence of $\lambda-A$.

Example 5. For the operators $A(V)$ defined in Example 5 the construction of Enss is

$$
M_{n}=1-\chi_{n}(x)
$$

on $L^{2}\left(R^{v}\right)$, where $\chi_{n}(x)=\chi(x / n)$ with $\chi \in C_{0}^{\infty}\left(R^{v}\right), 0 \leqq \chi \leqq 1$ and $\chi(x)=1$ for $|x|<1$. Then $M_{n}(x)$ has support in $\{|x|>n\}$ and Hypothesis 2 holds in the following form:

Lemma 3.2. Let $M_{n}$ be given by (3.3). Then the operators $A(V)=p^{2}+V(x)$ defined in Example 5 satisfy:

(i) If $\left\|A u_{m}\right\|$ is bounded for a sequence of unit vectors $u_{m} \in D(A)$ and $u_{m} \rightarrow \vec{w}$, then

$$
\lim _{m \rightarrow \infty}\left\|M_{n} u_{m}\right\|=1 \text { for all } n \text {. }
$$

(ii) For all $u \in D(A)$

$$
\left\|\left[M_{n}, A\right] u\right\| \leqq c n^{-1}(\|A u\|+\|u\|)
$$

with a constant $c$ independent of $V \in G$.

Proof. (i) $\chi_{n}\left(1+p^{2}\right)^{-1 / 2}$ is compact. Since the sequence $A u_{m}$ is bounded, the sequence $\left(1+p^{2}\right)^{1 / 2} u_{m}$ is bounded by (1.18) and thus weakly convergent to zero. Hence

$$
\left\|\chi_{n} u_{m}\right\|=\left\|\chi_{n}\left(1+p^{2}\right)^{-1 / 2}\left(1+p^{2}\right)^{1 / 2} u_{m}\right\| \rightarrow 0 \quad \text { as } \quad m \rightarrow \infty .
$$

(ii) $\left[M_{n}, A\right]=\left[p^{2}, \chi_{n}\right]=2 \operatorname{in}^{-1}(\nabla \chi(x / n)) \cdot p-n^{-2} \Delta \chi(x / n)$. By $\quad(1.18) \quad\left\|p_{k} u\right\|$ $\leqq$ const $(\|A u\|+\|u\|)$ with a constant independent of $V \in G$.

Remark. For any $\lambda \in W(A(V))$ there exists a characteristic sequence $\left\{u_{n}\right\}$ with $u_{n} \in C_{0}^{\infty}\left(R^{v}\right)$ and $u_{n}(x)=0$ for $|x|<n$. This follows from Lemma 3.2 and the fact that $C_{0}^{\infty}\left(R^{v}\right)$ is a core of $A(V)$. 
Theorem 3.3. Suppose that $A$ satisfies Hypothesis 2 and let

$$
d_{n}(\lambda)=\inf _{u \in D(A),\left\|M_{n} u\right\|=1}\left\|(\lambda-A) M_{n} u\right\| .
$$

If $\Omega$ is an open, connected complex set with $\Omega \cap \varrho(A) \neq \emptyset$ and $\lim _{n} \inf d_{n}(\lambda)>0$ for all $\lambda \in \Omega$, then $\Omega \cap \sigma_{\text {ess }}(A)=\emptyset$.

Proof. By Corollary 2.3 we need only show that $\Omega \cap W(A)=\emptyset$. Since $\lambda \in W(A)$ implies $\lim _{n \rightarrow \infty} d_{n}(\lambda)=0$ by Lemma 3.1, this follows from $\liminf _{n} d_{n}(\lambda)>0$.

Remark. A useful lower bound for $d_{n}(\lambda)$ is

$$
d_{n}(\lambda) \geqq \operatorname{dist}\left(\lambda, E_{n}\right),
$$

where

$$
E_{n}=\left\{\left(M_{n} u, A M_{n} u\right) \mid u \in D(A),\left\|M_{n} u\right\|=1\right\} .
$$

As an example we prove the first part of Theorem 1.1 which asserts that $\Omega \cap \sigma_{\text {ess }}\left(A\left(V_{0}\right)\right)=\emptyset$ :

Proof of Theorem 1.1 (first part). By (3.3) $M_{n}(x)$ has support in $\{|x|>n\}$. Since $E_{m}(V) \subset E_{n}(V)$ for $m>n$ we have

$$
d_{m}(\lambda) \geqq \operatorname{dist}\left(\lambda, E_{n}(V)\right)>0
$$

for all $\lambda \in \Omega$ and all $m>n$ by (1.20). Next we note that $E_{n}(V)+r \subset E_{n}(V)$ for any $r>0$. This follows from

$$
(u(a), A u(a))=(u, A u)+2 a \cdot(u, p u)+a^{2}(u, u),
$$

where $u(a)=u \exp (i a \cdot x), a \in R^{v}$. Without loss of generality we can therefore assume that $\Omega-r \subset \Omega$ for any $r>0$. Then $\Omega$ intersects $\varrho(A)$ in the complement of the sector (1.19). Hence $\Omega \cap \sigma_{\text {ess }}\left(A\left(V_{0}\right)\right)=\emptyset$ follows from Theorem 3.3.

\section{Invariance of the Essential Spectrum}

Here we apply Enss' construction to prove invariance theorems for $W(A)$ under additive perturbations. These results are not used in the following sections.

Theorem 4.1. Let $A$ and $B$ be closed operators on a Hilbert space $\mathscr{H}$ and suppose that $A$ satisfies Hypothesis 2. Then $W(A) \subset W(B)$ if one of the following conditions is satisfied:

(i) $(A-B) M_{n} u=K_{n}^{\prime} u+B_{n}^{\prime} u$ for all $u$ in a core of $A$, where $K_{n}^{\prime}$ is compact and $\left\|B_{n}^{\prime}\right\| \rightarrow 0$ as $n \rightarrow \infty$.

(ii) $\left[(z-A)^{-1}-(z-B)^{-1}\right] M_{n}=K_{n}^{\prime \prime}+B_{n}^{\prime \prime}$ for some $z \in \varrho(A) \cap \varrho(B)$, where $K_{n}^{\prime \prime}$ is compact and $\left\|B_{n}^{\prime \prime}\right\| \rightarrow 0$ as $n \rightarrow \infty$.

Remark. If $B$ also satisfies Hypothesis 2 with the same sequence $\left\{M_{n}\right\}$ then (i) or (ii) implies $W(A)=W(B)$.

Proof. (i) Let $\left\{u_{n}\right\}$ be a characteristic sequence of $\lambda-A$. By Lemma 3.1 we may choose a new characteristic sequence $v_{n}=M_{n} u_{m(n)}$ with $m(n)$ so large that 
$\left\|K_{n}^{\prime} u_{m}\right\|<n^{-1}$. Then $\left\|(A-B) v_{n}\right\|<\left\|B_{n}^{\prime}\right\|+n^{-1} \rightarrow 0$ as $n \rightarrow \infty$, so that $v_{n}$ is also a characteristic sequence of $\lambda-B$.

(ii) By the same argument we can choose $m(n)$ so large that $\left\|\left((z-A)^{-1}-(z-B)^{-1}\right) v_{n}\right\| \rightarrow 0$. Since $v_{n}$ is also a characteristic sequence of $(z-\lambda)^{-1}$ $-(z-A)^{-1}$ it follows that $\left.(z-\lambda)^{-1} \in W(z-B)^{-1}\right)$. By Lemma 2.4 this implies $\lambda \in W(B)$.

We first illustrate Theorem 4.1 with some known results for a pair of closed operators $A, B$ on $\mathscr{H}$ :

Example 6. (i) If $K$ is compact and $(A-B) u=K u$ for all $u \in D(A)=D(B)$, then $W(A)=W(B)$.

(ii) If $(z-A)^{-1}-(z-B)^{-1}$ is compact for some $z \in \varrho(A) \cap \varrho(B)$, then $W(A)$ $=W(B)$.

(iii) Let $\varrho(A) \neq \emptyset$ and $D\left(A^{m}\right) \subset D(B)$ for some integer $m>0$. If $A-B$ is $A^{m}$-compact, then $W(A) \subset W(B)$.

(iv) Let $D(A) \supset D(B), D\left(A^{2}\right) \subset D(B)$ and $\varrho(A) \cap \varrho(B) \neq \emptyset$. If $A-B$ is $A^{2}$-compact, then $W(A)=W(B)$.

Proof. (i) and (ii) are evident from Theorem 4.1 with $M_{n}=1$. (iii) Let $z \in \varrho(A)$, $M_{n}=(z-A)^{-m}$ for all $n$ and apply condition (i) of Theorem 4.1. (iv) $W(A) \subset W(B)$ follows from (iii). For the converse, note that

$$
\left[(z-A)^{-1}-(z-B)^{-1}\right](z-B)^{-1}=(z-B)^{-1} \underbrace{(A-B)(z-A)^{-2}}_{\text {compact }} \underbrace{(z-A)(z-B)^{-1}}_{\text {bounded }}
$$

for some $z \in \varrho(A) \cap \varrho(B)$. Now use condition (ii) of Theorem 4.1 with $M_{n}=(z-B)^{-1}$.

On this abstract level the possibilities for constructing useful sequences $\left\{M_{n}\right\}$ are rather limited. We can do better in concrete situations:

Example 7. On $L^{2}\left(R^{v}\right)$ we consider the formal operator

$$
A=Q_{0}(p)+Q_{1}(p) V(x) Q_{2}(p)
$$

We assume:

(i) The $Q_{i}$ are polynomials on $R^{v}$,

(ii) $V \in L_{\text {loc }}^{1}\left(R^{v}\right)$, and the form $t$ defined on $\mathscr{S}\left(R^{v}\right)$ by

$$
t(u, v)=\left(u, Q_{0} v\right)+\left(Q_{1}^{*} u, V Q_{2} v\right)
$$

is sectorial and closable. $A$ is defined by the closure $\bar{t}$ of $t$ via the representation theorem [8, Chap. VI]

$$
(u, f(p) u) \leqq a|t(u, u)|+b(u, u)
$$

for all $u \in \mathscr{S}\left(R^{v}\right)$, where $f$ is a real measurable function on $R^{v}$ with $f(x) \rightarrow \infty$ as $|x| \rightarrow \infty$.

(iv) Let $m_{i}$ be the smallest integer such that $2 m_{i} \geqq$ degree of $Q_{i} . V(x)$ and its derivatives up to order $\max \left(2 m_{1}, 2 m_{2}\right)$ are continuous on $R \leqq|x|<\infty$ and vanish as $|x| \rightarrow \infty$. 
Theorem 4.2. Under the conditions (i)-(iv) given above, $W(A)=W\left(Q_{0}\right)$.

Remarks. $W\left(Q_{0}\right)=\sigma_{\text {ess }}\left(Q_{0}\right)$ is the range of the function $x \rightarrow Q_{0}(x)$ on $R^{v}$. If $C \backslash W\left(Q_{0}\right)$ is connected, it follows from Corollary 2.3 that $\sigma_{\text {ess }}(A)=W(A)=\sigma_{\text {ess }}\left(Q_{0}\right)$.

More general operators of this type are constructed and discussed by Schechter [12]. Our invariance result is considerably stronger than this Theorem 10.2 in the sense that we impose no restrictions on the degrees of $Q_{1}$ and $Q_{2}$.

Proof. We choose

$$
M_{n}=M=(1-\chi(x))\left(1+p^{2}\right)^{-r}(1-\chi(x))
$$

for all $n$, with $r>0$ and real $\chi \in C_{0}^{\infty}\left(R^{v}\right)$ to be adjusted in the course of the proof. First we verify Hypothesis 2 . Since $M-\left(1+p^{2}\right)^{-r}$ is compact, $u_{n} \underset{w}{\rightarrow} 0$ implies

$$
\lim _{n \rightarrow \infty}\left\|M u_{n}-\left(1+p^{2}\right)^{-r} u_{n}\right\|=0 .
$$

On the other hand it follows from $\left\|u_{n}\right\|=1$ and $\left(u_{n}, f(p) u_{n}\right)<c$ that

$$
\begin{aligned}
\left\|\left(1+p^{2}\right)^{-r} u_{n}\right\|^{2} & \geqq\left(1+d^{2}\right)^{-2 r}\left(1-\int_{|k|>d} d^{v} k|\hat{u}(k)|^{2}\right) \\
& \geqq\left(1+d^{2}\right)^{-2 r}\left[1-c\left(\inf _{|k|>d} f(k)\right)^{-1}\right]>a>0
\end{aligned}
$$

for sufficiently large $d$ depending only on $c$, where $\hat{u}$ is the Fourier transform of $u$. It follows from this and (4.2) that (3.1) is satisfied. To prove (3.2) we first choose $2 r \geqq$ degree of $Q_{0}$. Then

$$
Q_{0} M u=Q_{0}\left(1+p^{2}\right)^{-r} u+K_{1} u
$$

and

$$
Q_{0}^{*} M u=Q_{0}^{*}\left(1+p^{2}\right)^{-r} u+K_{2}^{*} u
$$

for all $u \in \mathscr{S}\left(R^{v}\right)$ with $K_{1}$ and $K_{2}$ compact. Now we choose $\chi$ such that $\chi(x)=1$ for $|x|<R$ and we set $V_{\chi}=V(1-\chi)$. Observing that $Q_{2}$ is a local operator we then find for all $u \in \mathscr{S}\left(R^{v}\right)$ :

$$
\begin{aligned}
Q_{1} V Q_{2} M u= & Q_{1} V_{\chi} Q_{2} M u \\
= & {\left[Q_{1}\left(1+p^{2}\right)^{-m_{1}}\right]\left[\left(1+p^{2}\right)^{m_{1}} V_{\chi}\left(1+p^{2}\right)^{-r+m_{2}}\right] } \\
& \cdot\left[Q_{2}\left(1+p^{2}\right)^{-m_{2}}\right]\left[\left(1+p^{2}\right)^{r}(1-\chi)\left(1+p^{2}\right)^{-r}(1-\chi)\right] u \\
= & K_{3} u .
\end{aligned}
$$

Choosing now $r>m_{1}+m_{2}$, all four factors in square brackets are bounded and the second one is compact, hence $K_{3}$ is compact. Similarly, $Q_{2}^{*} V^{*} Q_{1}^{*} M u=K_{4}^{*} u$ for all $u \in \mathscr{S}\left(R^{v}\right)$ with $K_{4}$ compact. Therefore

$$
\bar{t}(u, M v)-\bar{t}(M u, v)=(u, K v)
$$

for all $u, v \in \mathscr{S}\left(R^{v}\right)$ with $K=K_{1}-K_{2}+K_{3}-K_{4}$ compact, and

$$
|t(M u, M u)| \leqq\|M\|\left(\left\|Q_{0}\left(1+p^{2}\right)^{-r}\right\|+\left\|K_{1}\right\|+\left\|K_{3}\right\|\right)(u, u) .
$$

Therefore $M$ is a bounded operator from $\mathscr{S}\left(R^{v}\right)$ into the form domain $D(t)$ (equipped with $t$-norm), and as a consequence (4.3) extends by continuity to all 
$u \in D(\bar{t})$. It follows from the representation theorem that $M v \in D(A)$ and $[A, M] v=K v$. Therefore Hypothesis 2 is satisfied with $B_{n}=0$ and $K_{n}=K$. To apply Theorem 4.1 we remark that

$$
\left(A-Q_{0}\right) M u=K_{3} u
$$

for all $u \in D(A)$, which proves $W(A) \subset W\left(Q_{0}\right)$. To show the converse we note that $\mathscr{S}\left(R^{v}\right)$ is a core of $Q_{0}$ and that $\left[Q_{0}, M\right] u=\left(K_{1}-K_{2}\right) u$ for all $u \in \mathscr{S}\left(R^{v}\right)$. Since (4.4) also holds for $u \in \mathscr{S}\left(R^{v}\right)$, it follows that $W\left(Q_{0}\right) \subset W(A)$.

\section{Stability of Discrete Eigenvalues}

We now continue the discussion of stability as outlined in the introduction.

Lemma 5.1. Suppose that $A(\kappa)$ satisfies Hypothesis 1 and that $z \notin \sigma_{\mathrm{ess}}(A(\kappa))$ for $\kappa$ close to $\kappa_{0}$. If $z \notin \sigma_{p}\left(A\left(\kappa_{0}\right)\right)$, then $z \in \Delta$ unless there exist sequences $\kappa_{n} \in G$ and $u_{n} \in \mathscr{H}$ such that

$$
\begin{gathered}
\kappa_{n} \rightarrow \kappa_{0}, \\
u_{n} \in D\left(A\left(\kappa_{n}\right)\right),\left\|u_{n}\right\| \nrightarrow 0, u_{n} \rightarrow 0 \text { and }\left\|\left(z-A\left(\kappa_{n}\right)\right) u_{n}\right\| \rightarrow 0 .
\end{gathered}
$$

Proof. Suppose that $\|(z-A(\kappa)) u\| \geqq \varepsilon\|u\|, \varepsilon>0$, for $\kappa$ close to $\kappa_{0}$ and all $u \in D(A(\kappa))$. Since $z \notin \sigma_{\text {ess }}(A(\kappa))$, this implies $z \in \varrho(A(\kappa))$ and $\|R(z, \kappa)\| \leqq \varepsilon^{-1}$, i.e. $z \in \Delta$. Therefore, if $z \notin \Delta$, there exist sequences $\kappa_{n} \rightarrow \kappa_{0}, \quad u_{n} \in D\left(A\left(\kappa_{n}\right)\right), \quad\left\|u_{n}\right\|=1$ such that $\left\|\left(z-A\left(\kappa_{n}\right)\right) u_{n}\right\| \rightarrow 0$. By passing to a subsequence we may assume $u_{n} \rightarrow \vec{w} u$. It then follows from Hypothesis 1 that

$$
\begin{aligned}
0 & =\lim _{n \rightarrow \infty}\left(v,\left(z-A\left(\kappa_{n}\right)\right) u_{n}\right) \\
& =\lim _{n \rightarrow \infty}\left(\left(z-A\left(\kappa_{n}\right)\right)^{*} v, u_{n}\right) \\
& =\left(\left(z-A\left(\kappa_{0}\right)\right)^{*} v, u\right)
\end{aligned}
$$

for all $v \in D^{*}$. Since $D^{*}$ is a core of $A^{*}\left(\kappa_{0}\right)$, it follows that $u \in D\left(A\left(\kappa_{0}\right)\right)$ and $\left(z-A\left(\kappa_{0}\right)\right) u=0$. Hence $u=0$ since $z \notin \sigma_{p}\left(A\left(\kappa_{0}\right)\right)$ by hypothesis.

To prove (1.25) we want to exclude (5.1) for $z$ close to $\lambda$. This is done by applying Enss' construction to sequences of type (5.1), using the following analogue of Hypothesis 2:

Hypothesis 3. Suppose that $A(\kappa)$ satisfies Hypothesis 1 and that $\Delta \neq \emptyset$. Let $\left\{M_{n}(\kappa)\right\}$ be a sequence of families of operators on $G$ with the following properties:

(i) $M_{n}(\kappa)$ is bounded uniformly in $n$ and $\kappa$.

(ii) If $\left\{\kappa_{m}\right\} \subset G$ and $\left\{u_{m}\right\} \subset \mathscr{H}$ are sequences satisfying

$$
\begin{gathered}
\kappa_{m} \rightarrow \kappa_{0}, \\
u_{m} \in D\left(A\left(\kappa_{m}\right)\right),\left\|u_{m}\right\| \nrightarrow 0, u_{m} \rightarrow \underset{w}{ } 0 \text { and } \\
\left\|A\left(\kappa_{m}\right) u_{m}\right\| \leqq \text { const for all } m,
\end{gathered}
$$


then $\limsup _{m}\left\|M_{n}\left(\kappa_{m}\right) u_{m}\right\|>a>0$ for all $n$.

(iii) $M_{n}(\kappa)$ maps $D(A(\kappa))$ into itself and

$$
\left[M_{n}(\kappa), A(\kappa)\right] u=B_{n}(\kappa) u+K_{n}(\kappa) u
$$

for all $u \in D(A(\kappa))$. For some $z \in \Delta$,

$$
\lim _{\kappa \rightarrow \kappa_{0}} K_{n}(\kappa) R(z, \kappa)=K_{n}\left(\kappa_{0}\right) R\left(z, \kappa_{0}\right)
$$

exists in norm and is compact, and

$$
\lim _{n \rightarrow \infty}\left\|B_{n}(\kappa) R(z, \kappa)\right\|=0
$$

uniformly in $\kappa$.

Lemma 5.2. If Hypothesis 3 holds for some $z_{0} \in \Delta$, it holds for all $z \in \Delta$. Given any compact $\Gamma \subset \Delta$ we have

$$
\lim _{\kappa \rightarrow \kappa_{0}}\left\|K_{n}(\kappa) R(z, \kappa)-K_{n}\left(\kappa_{0}\right) R\left(z, \kappa_{0}\right)\right\|=0
$$

uniformly in $z \in \Gamma$, and

$$
\lim _{n \rightarrow \infty}\left\|B_{n}(\kappa) R(z, \kappa)\right\|=0
$$

uniformly in $(z, \kappa) \in \Gamma \times U$, where $U$ is some neighbourhood of $\kappa_{0}$.

Proof. Consider the identity

$$
K_{n}(\kappa) R(z, \kappa)=K_{n}(\kappa) R\left(z_{0}, \kappa\right)+\left(z-z_{0}\right) K_{n}(\kappa) R\left(z_{0}, \kappa\right) R(z, \kappa) .
$$

For $\kappa \rightarrow \kappa_{0}$ we have $K_{n}(\kappa) R\left(z_{0}, \kappa\right) \rightarrow K_{n}\left(\kappa_{0}\right) R\left(z_{0}, \kappa_{0}\right)$ in norm, and $R^{*}(z, \kappa) \rightarrow R^{*}\left(z, \kappa_{0}\right)$ strongly by Lemma 1.2. Since $K_{n}\left(\kappa_{0}\right) R\left(z_{0}, \kappa_{0}\right)$ is compact, it follows that the last term in (5.5) converges in norm to its value at $\kappa_{0}$. Local uniformity in $z$ follows from (1.8). To prove (5.4) let $U$ be a neighbourhood of $\kappa_{0}$ such that

$$
\lim _{n \rightarrow \infty}\left\|B_{n}(\kappa) R\left(z_{0}, \kappa\right)\right\|=0
$$

uniformly in $\kappa \in U$ and $\|R(z, \kappa)\| \leqq M<\infty$ for all $(z, \kappa) \in \Gamma \times U$. Using (5.5) with $B_{n}(\kappa)$ in place of $K_{n}(\kappa)$ we find for the last term

$$
\left\|B_{n}(\kappa) R\left(z_{0}, \kappa\right) R(z, \kappa)\right\| \leqq M\left\|B_{n}(\kappa) R\left(z_{0}, \kappa\right)\right\| \rightarrow 0
$$

uniformly in $(z, \kappa) \in \Gamma \times U$.

By following the proof of Lemma 3.1 it is straightforward to obtain the analogous

Lemma 5.3. Let $A(\kappa)$ satisfy Hypothesis 3. If $\left\{\kappa_{n}, u_{n}\right\}$ are sequences satisfying (5.1), then so are $\left\{\kappa_{m}, v_{n}=M_{n}\left(\kappa_{m}\right) u_{m}\right\}$ provided that $m=m(n)$ is chosen sufficiently large for each $n$.

Theorem 5.4. Suppose that A( $\kappa)$ satisfies Hypothesis 3 and let

$$
d_{n}(\lambda, \kappa)=\inf _{\substack{u \in D(A(\kappa)) \\\left\|M_{n}(\kappa) u\right\|=1}}\left\|(\lambda-A(\kappa)) M_{n}(\kappa) u\right\| .
$$


Let $\lambda \in C$ be such that for some $n_{0}$ and some $\delta>0$

$$
\operatorname{dist}\left\{\lambda, \sigma_{\text {ess }}(A(\kappa))\right\}>\delta
$$

and

$$
d_{n}(\lambda, \kappa)>\delta
$$

for all $n>n_{0}$ and all $\kappa$ in some neighbourhood of $\kappa_{0}$. Then the following alternative holds :

(i) If $\lambda \notin \sigma_{p}\left(A\left(\kappa_{0}\right)\right)$, then $\lambda \in \Delta$.

(ii) If $\lambda \in \sigma_{p}\left(A\left(\kappa_{0}\right)\right)$, then $\lambda$ is a stable eigenvalue with respect to the family $A(\kappa)$.

Combining this result with Theorem 3.3 we obtain:

Theorem 5.5. Suppose that $A(\kappa)$ satisfies Hypothesis 3 for each $\kappa_{0} \in G$. Let $\Omega$ be an open, connected complex set such that $\Omega \cap \varrho(A(\kappa)) \neq \emptyset$ and $d_{n}(\lambda, \kappa) \geqq \delta>0$ for all $\kappa \in G$, $\lambda \in \Omega$, and $n>n_{0}$. Then $A(\kappa)$ has purely discrete spectrum in $\Omega$ and each eigenvalue $\lambda \in \Omega$ of $A(\kappa)$ is stable with respect to variations of $\kappa$.

For a first illustration we return to Example 5:

Proof of Theorem 1.1 (conclusion). Let $M_{n}$ be given by (3.3) for all values of the perturbation parameter $V$. Inspecting the proof of Lemma 3.2 we see that $A(V)$ satisfies Hypothesis 3 for any $V_{0} \in G$. The other conditions of Theorem 5.5 have already been verified in the first part of the proof (Sect. 3).

Proof of Theorem 5.4. (i) Let $\lambda \notin \sigma_{p}\left(A\left(\kappa_{0}\right)\right)$. If $\lambda \notin \Delta$, there exist sequences $\left\{\kappa_{n}, u_{n}\right\}$ satisfying (5.1). It then follows from Lemma 5.3 that $d_{n}\left(\lambda, \kappa_{m(n)}\right) \rightarrow 0$ as $n \rightarrow \infty$ in contradiction to (5.8). Hence $\lambda \in \Delta$.

(ii) Let $\lambda \in \sigma_{p}\left(A\left(\kappa_{0}\right)\right)$. If $|z-\lambda|>0$ is sufficiently small, then $z \notin \sigma_{p}\left(A\left(\kappa_{0}\right)\right)$, $z \notin \sigma_{\text {ess }}(A(\kappa))$ and $d_{n}(z, \kappa) \geqq \delta / 2$ for all $n>n_{0}$ and all $\kappa$ in some neighbourhood of $\kappa_{0}$. By part (i) of the proof it follows that $z \in \Delta$. This proves (1.25)-(1.27). Suppose now that (1.28) does not hold. Then there exist sequences $\kappa_{m} \rightarrow \kappa_{0}$ and $u_{m} \in \mathscr{H},\left\|u_{m}\right\|=1$, such that

$$
P\left(\kappa_{m}\right) u_{m}=u_{m} \quad \text { and } \quad P\left(\kappa_{0}\right) u_{m}=0
$$

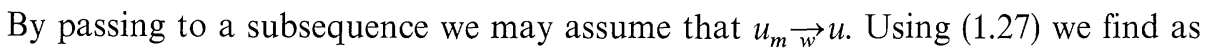
weak limits of (5.9):

$$
P\left(\kappa_{0}\right) u=u \quad \text { and } \quad P\left(\kappa_{0}\right) u=0 \text {, }
$$

which shows that $u_{m} \rightarrow \vec{w}$. In (1.10) we now fix the radius $r$ of the circle $\Gamma_{r}$ in the interval $0<r<\delta / 2$. Then by (5.8)

$$
d_{n}\left(z, \kappa_{m}\right) \geqq d_{n}\left(\lambda, \kappa_{m}\right)-r \geqq \delta / 2
$$

for all $z \in \Gamma_{r}$ and sufficiently large $n, m$. Let $v_{m}(z)=R\left(z, \kappa_{m}\right) u_{m}$. Then by (5.10) and Hypothesis 3

$$
\begin{aligned}
(\delta / 2)\left\|M_{n}\left(\kappa_{m}\right) v_{m}\right\| & \leqq\left\|\left(z-A\left(\kappa_{m}\right)\right) M_{n}\left(\kappa_{m}\right) v_{m}\right\| \\
& \leqq\left\|M_{n}\left(\kappa_{m}\right) u_{m}\right\|+\left\|K_{n}\left(\kappa_{m}\right) R\left(z, \kappa_{m}\right) u_{m}\right\|+\left\|B_{n}\left(\kappa_{m}\right) R\left(z, \kappa_{m}\right) u_{m}\right\|
\end{aligned}
$$


for all $z \in \Gamma_{r}$ and sufficiently large $n, m$. Operating with $(\delta / 2) M_{n}\left(\kappa_{m}\right)$ on

$$
u_{m}=P\left(\kappa_{m}\right) u_{m}=(2 \pi i)^{-1} \oint_{\Gamma_{r}} d z v_{m}(z)
$$

we thus find the estimate

$$
\begin{aligned}
(\delta / 2)\left\|M_{n}\left(\kappa_{m}\right) u_{m}\right\| \leqq & \left\|M_{n}\left(\kappa_{m}\right) u_{m}\right\| \\
& +(2 \pi)^{-1} \oint_{I_{r}}|d z|\left\|K_{n}\left(\kappa_{m}\right) R\left(z, \kappa_{m}\right) u_{m}\right\| \\
& +(2 \pi)^{-1} \oint_{\Gamma_{r}}|d z|\left\|B_{n}\left(\kappa_{m}\right) R\left(z, \kappa_{m}\right) u_{m}\right\| .
\end{aligned}
$$

Since $u_{m} \rightarrow \vec{w}$, it follows from (5.3) that the first integral vanishes for each $n$ as $m \rightarrow \infty$, while the second one vanishes uniformly in $m$ as $n \rightarrow \infty$ by (5.4). Since $r<\delta / 2$ this implies

$$
\lim _{n \rightarrow \infty} \lim _{m} \sup _{\|}\left\|M_{n}\left(\kappa_{m}\right) u_{m}\right\|=0 .
$$

On the other hand, the sequence $A\left(\kappa_{m}\right) u_{m}=A\left(\kappa_{m}\right) P\left(\kappa_{m}\right) u_{m}$ is bounded since $A(\kappa) P(\kappa)$ is bounded uniformly for $\kappa$ close to $\kappa_{0}$ by part (i) of the proof. Therefore (5.11) is in contradiction to Hypothesis 3 (ii).

Example 8 (Magnetic fields).

$$
A(a)=(p-a(x))^{2}+V(x)
$$

on $L^{2}\left(R^{v}\right)$. We consider a fixed potential $V$ satisfying the hypothesis of Theorem 6.1, and we restrict the perturbation parameter $a$ to the space $C^{1}$ of real $C^{1}$-vector fields on $R^{v}$ with the topology given by the seminorms

$$
\|a\|_{r}=\sup _{\substack{|x| \leq r \\ i, k=1 \ldots \nu}}\left(\left|a_{i}(x)\right|+\left|\frac{\partial a_{i}}{\partial x_{k}}(x)\right|\right), \quad r=1,2,3, \ldots
$$

$A(a)$ is then constructed as the closure of (5.12), defined on $C_{0}^{\infty}\left(R^{v}\right)$ (Theorem 6.2). It satisfies Hypothesis 1 for any $a_{0} \in C^{1}$. The analogue to Theorem 1.1 is

Theorem 5.6. Let $A(a)$ be the family defined in Example 8, and

$$
E_{n}(a)=\left\{(u, A(a) u) \mid u \in C_{0}^{\infty}\left(R^{v}\right),\|u\|=1 \text { and } u(x)=0 \text { for }|x| \leqq n\right\} .
$$

Let $\Omega$ be an open, connected complex set and $G_{\Omega}$ a subset of vector fields $a \in C^{1}$ such that $\Omega \cap E_{n}(a)=\emptyset$ for some fixed $n$ and all $a \in G_{\Omega}$. Then for each $a_{0} \in G_{\Omega}$, the spectrum of $A\left(a_{0}\right)$ in $\Omega$ is purely discrete and each eigenvalue $\lambda \in \Omega$ of $A\left(a_{0}\right)$ is stable with respect to the family $\left\{A(a) \mid a \in G_{\Omega}\right\}$.

Example 4. Let $A(\kappa)$ be given by (1.6). If $u \in C_{0}^{\infty}\left(R^{3}\right),\|u\|=1$ and $u(x)=0$ for $|x| \leqq n$, then $(u, A(\kappa) u)$ has distance less than $1 / n$ from $\sigma\left((p-\kappa \wedge x)^{2}\right)=[|\kappa|, \infty)$. Hence $A(\kappa)$ has purely discrete spectrum in the complement of $[|\kappa|, \infty)$ and each one of these eigenvalues is stable with respect to variations of $\kappa \in R^{3}$.

Proof of Theorem 5.6. Defining $M_{n}$ by (3.3) and using the bound (6.9) on $\left(u, \pi^{2} u\right)$ the commutator estimate (3.5) is seen to hold uniformly in $a \in C^{1}$. It remains to 
establish Hypothesis 3(ii). Suppose that $a_{n} \in C^{1}$ and $u_{n} \in C_{0}^{\infty}\left(R^{v}\right)$ are sequences satisfying $a_{n} \rightarrow a_{0}, u_{n} \rightarrow w_{w} 0$ and $\left\|A\left(a_{n}\right) u_{n}\right\|<$ const for all $n$. We have to show that $\left\|\chi u_{n}\right\| \rightarrow 0$ for any $\chi \in C_{0}^{\infty}\left(R^{v}\right)$. By (6.9) $\left\|\pi_{k} u_{n}\right\|$ is bounded uniformly in $n$ for each $k=1 \ldots \nu$. The same follows successively for $\left\|\chi \pi_{k} u_{n}\right\|,\left\|\pi_{k} \chi u_{n}\right\|$, and $\left\|p_{k} \chi u_{n}\right\|$ since $a_{n} \rightarrow a_{0}$ uniformly in $x$. Let $\Lambda \in C_{0}^{\infty}\left(R^{v}\right)$ such that $\Lambda \chi=\chi$. Then $\left\|\chi u_{n}\right\|$ $=\left\|\Lambda\left(1+p^{2}\right)^{-1 / 2}\left(1+p^{2}\right)^{1 / 2} \chi u_{n}\right\| \rightarrow 0$ as $n \rightarrow \infty$ since $\Lambda\left(1+p^{2}\right)^{-1 / 2}$ is compact and $\left(1+p^{2}\right)^{1 / 2} u_{n} \rightarrow$. As a result, $A(a)$ satisfies Hypothesis 3 for each $a_{0} \in C^{1}$. Theorem 5.6 now follows from Theorem 5.5.

Concluding Remarks. For simplicity we have chosen our examples from the class of "one-body" problems, in which the unperturbed potential $V(x)$ has a simple behavior for $|x| \rightarrow \infty$ [e.g. $V(x) \rightarrow 0$ or $V(x) \rightarrow \infty$ ]. Actually the construction of Enss [5] was devised to deal with $N$-body systems, i.e. with potentials whose behavior depends strongly on the direction in which $x \rightarrow \infty$ in $R^{v}$. An abstract version of it is given by the following extension of Theorem 3.3 :

Theorem 5.7. Suppose that Hypothesis 2 holds for $A$ and

$$
M_{n}=\sum_{\alpha=1}^{p} M_{n}^{\alpha} \quad(p<\infty),
$$

where the operators $M_{n}^{\alpha}$ are bounded uniformly in $n, \alpha$ and satisfy (3.2) for each $\alpha$. Let

$$
d_{n}^{\alpha}(\lambda)=\inf _{\substack{u \in D(A) \\\left\|M_{n}^{\alpha} u\right\|=1}}\left\|(\lambda-A) M_{n}^{\alpha} u\right\| .
$$

If $\Omega$ is an open, connected complex set with $\Omega \cap \varrho(A)=\emptyset$ and $\liminf _{n} d_{n}^{\alpha}(\lambda)>0$ for all $\alpha$ and all $\lambda \in \Omega$, then $\Omega \cap \sigma_{\mathrm{ess}}(A)=\emptyset$.

Proof. Suppose that

$$
\limsup _{m}\left\|M_{n} u_{m}\right\|>a>0
$$

for some sequence $\left\{u_{m}\right\}$ and all $n$. Then

$$
\limsup _{m}\left\|M_{n}^{\alpha} u_{m}\right\|>a p^{-1}>0
$$

for each $n$ and some $\alpha=\alpha(n)$. By passing to an infinite subsequence of $\left\{M_{n}\right\}$ we may therefore assume that (5.15) holds for some fixed $\alpha$ and all $n$. With this remark the proofs of Lemma 3.1 and Theorem 3.3 are immediately extended to Theorem 5.7.

By the same argument Theorem 5.4 extends to

Theorem 5.8. Suppose that Hypothesis 3 holds for $A(\kappa)$ and

$$
M_{n}(\kappa)=\sum_{\alpha=1}^{p} M_{n}^{\alpha}(\kappa) \quad(p<\infty),
$$

where the operators $M_{n}^{\alpha}(\kappa)$ are bounded uniformly in $n, \alpha, \kappa$ and satisfy the commutator condition (iii) of Hypothesis 3 for each $\alpha$. Then Theorem 5.4 holds if (5.8) is 
replaced by the condition

$$
d_{n}^{\alpha}(\lambda, \kappa)=\inf _{\substack{u \in D(A(\kappa)) \\\left\|M_{n}^{\alpha}(\kappa) u\right\|=1}}\left\|(\lambda-A(\kappa)) M_{n}^{\alpha}(\kappa) u\right\|>\delta>0
$$

for all $\alpha$, all $n>n_{0}$ and all $\kappa$ in some neighbourhood of $\kappa_{0}$.

Typically, $M_{n}^{\alpha}(\kappa)$ is constructed to have a range on which $A(\kappa)$ reduces to a simpler operator $A^{\alpha}(\kappa)$ [up to an error of order $o(n)$ ]. Then the crucial estimate (5.16) reduces to the corresponding estimate for $A^{\alpha}(\kappa)$, which may be obtained from the numerical range of $A^{\alpha}(\kappa)$ or from an upper bound on $\left\|\left(\lambda-A^{\alpha}(\kappa)\right)^{-1}\right\|$. Examples where stability can be proved in this way are the Stark and Zeeman effect for $N$-electron atoms $[7,4]$. A similar but formally different case is the cluster limit $|\kappa| \rightarrow \infty$ in Example 2 (or in more general systems [9]), where $A(\kappa)$ reduces to $p^{2}-|x|^{-1}$ or $p^{2}-|x-\kappa|^{-1}$ in complementary regions of $R^{3}$ with an error of order $|\kappa|^{-1}$.

As a general conclusion we may say that discrete eigenvalues of Schrödinger operators on $L^{2}\left(R^{v}\right)$ are stable under large classes of perturbations (Hypothesis 1) as long as they remain isolated from the essential spectrum, which in turn is governed by the behavior of the operator near infinity in $R^{v}$. Weyl's criterion and the construction of Enss provide a relatively simple, direct method to deal with the qualitative question of stability. It is clear, however, that resolvent equations and resolvent estimates are still needed for the quantitative and computational aspects of perturbation theory.

\section{Auxiliary Results}

In Example 5 we have implicitly defined a space $G$ of potentials $V \in L_{\text {loc }}^{2}\left(R^{v}\right)$. The following theorem gives explicit sufficient conditions for $V \in G$ :

Theorem 6.1. Let $\alpha, \beta, \gamma$ be real with $|\gamma|<\pi / 2$ and $0 \leqq \alpha<\cos \gamma$. Suppose that $V=V_{1}+V_{2}$ in $L_{\text {loc }}^{2}\left(R^{v}\right)$ such that

(i) $\cos \gamma \operatorname{Re} V_{1}(x)+\sin \gamma \operatorname{Im} V_{1}(x) \geqq 0$ (a.e.) and

(ii) $\left\|V_{2} u\right\| \leqq \alpha\left\|p^{2} u\right\|+\beta\|u\|$ for all $u \in C_{0}^{\infty}\left(R^{v}\right)$.

On $L^{2}\left(R^{v}\right)$ we consider the operator $A_{0}=p^{2}+V$ with domain $C_{0}^{\infty}\left(R^{v}\right)$. Then there exist constants $\xi, \eta$ depending only on $\alpha, \beta, \gamma$ such that

$$
\left(u, p^{2} u\right) \leqq \xi\left\{\cos \gamma \operatorname{Re}\left(u, A_{0} u\right)+\sin \gamma \operatorname{Im}\left(u, A_{0} u\right)+\eta(u, u)\right\}
$$

for all $u \in C_{0}^{\infty}\left(R^{v}\right)$.

(iii) Suppose further that for any $\chi \in C_{0}^{\infty}\left(R^{v}\right) V_{1} \chi$ has arbitrarily small relative bound with respect to $p^{2}$.

Then $\operatorname{Ran}\left(z+A_{0}\right)$ is dense in $L^{2}\left(R^{v}\right)$ for sufficiently large real $z$.

Remark. Apart from hypothesis (iii) this is a natural extension of Theorem X.29 in [10] to the non-selfadjoint case. We suspect that it holds without the extra assumption (iii) as it does in the case $v \leqq 3$, where (iii) follows from $V_{1} \in L_{\mathrm{loc}}^{2}\left(R^{v}\right)$.

Proof. We first prove (6.1). For the quadratic forms on $C_{0}^{\infty}\left(R^{v}\right)$ we have

$$
\operatorname{Re} V_{1}=\operatorname{Re} A_{0}-p^{2}-\operatorname{Re} V_{2} ; \quad \operatorname{Im} V_{1}=\operatorname{Im} A_{0}-\operatorname{Im} V_{2} .
$$


Inserting this into hypothesis (i) we obtain

$$
\begin{aligned}
\cos \gamma \operatorname{Re} A_{0}+\sin \gamma \operatorname{Im} A_{0} & \geqq \cos \gamma p^{2}+\cos \gamma \operatorname{Re} V_{2}+\sin \gamma \operatorname{Im} V_{2} \\
& \geqq \cos \gamma p^{2}-\left|V_{2}\right| .
\end{aligned}
$$

Choosing $\delta$ in $\alpha<\delta<\cos \gamma$ we have

$$
\left\|V_{2} u\right\| \leqq \alpha \delta^{-1}\left\|\delta p^{2} u\right\|+\beta\|u\|
$$

Since $\alpha \delta^{-1}<1$ this implies

$$
\delta p^{2}-\left|V_{2}\right| \geqq-\beta \delta(\delta-\alpha)^{-1} .
$$

Inserting this into (6.2) we find the desired estimate

$$
p^{2} \leqq(\cos \gamma-\delta)^{-1}\left\{\cos \gamma \operatorname{Re} A_{0}+\sin \gamma \operatorname{Im} A_{0}+\beta \delta(\delta-\alpha)^{-1}\right\} .
$$

As a consequence of (6.1) $A_{0}$ has numerical range in the halfplane

$$
S=\{x+i y \mid x \cos \gamma+y \sin \gamma+\eta \geqq 0\},
$$

which implies

$$
\left\|\left(z-A_{0}\right) u\right\| \geqq\|u\| \operatorname{dist}(z, S)
$$

We now fix $z \notin S$ and prove that $\operatorname{Ran}\left(z-A_{0}\right)$ is dense. Given any $f \in C_{0}^{\infty}\left(R^{v}\right)$ and $\varepsilon>0$ we construct $g \in C_{0}^{\infty}\left(R^{v}\right)$ such that

$$
\left\|\left(z-A_{0}\right) g-f\right\|<\varepsilon .
$$

For any $\chi \in C_{0}^{\infty}\left(R^{v}\right)$ with $0 \leqq \chi \leqq 1$ we define $A_{\chi}=p^{2}+V_{\chi}, V_{\chi}=V_{1} \chi+V_{2}$. Since $V_{1} \chi$ also satisfies hypothesis (i) it follows that (6.1) and (6.2) hold for $A_{\chi}$ with constants $\xi, \eta$ independent of $\chi$. By hypothesis (iii) $V_{\chi}$ has relative bound less than 1 with respect to $p^{2}$. Therefore $A_{\chi}$ has a nonempty resolvent set containing some left halfplane, so that (6.3) implies

$$
\left\|\left(z-A_{\chi}\right)^{-1}\right\| \leqq[\operatorname{dist}(z, S)]^{-1}
$$

for all $z \notin S$ and all $\chi$. Since $C_{0}^{\infty}\left(R^{v}\right)$ is a core of $p^{2}$ and thus of $A_{\chi}$ we can find $h_{\chi} \in C_{0}^{\infty}\left(R^{v}\right)$ such that

$$
\left\|\left(z-A_{\chi}\right) h_{\chi}-f\right\|<\varepsilon / 2
$$

which by (6.5) implies

$$
\left\|h_{\chi}\right\| \leqq(\|f\|+\varepsilon / 2)[\operatorname{dist}(z, S)]^{-1} .
$$

Using (6.1) we then obtain a uniform estimate

$$
\left(h_{\chi},\left(1+p^{2}\right) h_{\chi}\right) \leqq c^{2}
$$

with $c$ independent of the choice of $\chi$ and $h_{\chi}$. Now let $\Lambda \in C_{0}^{\infty}\left(R^{v}\right), 0 \leqq \Lambda \leqq 1$, such that $\Lambda f=f$ and

$$
\left\|\left[p^{2}, \Lambda\right]\left(1+p^{2}\right)^{-1 / 2}\right\|<\varepsilon / 2 c .
$$


Adjusting $\chi$ such that $\chi \Lambda=\Lambda$ it follows from (6.6) that

$$
\begin{aligned}
\varepsilon / 2 & \geqq\left\|\Lambda\left(\left(z-A_{\chi}\right) h_{\chi}-f\right)\right\|=\left\|\left(z-V_{1}-V_{2}\right) \Lambda h_{\chi}-\Lambda p^{2} h_{\chi}-f\right\| \\
& \geqq\left\|\left(z-A_{0}\right) \Lambda h_{\chi}-f\right\|-\left\|\left[p^{2}, \Lambda\right]\left(1+p^{2}\right)^{-1 / 2}\right\|\left\|\left(1+p^{2}\right)^{1 / 2} h_{\chi}\right\| .
\end{aligned}
$$

From (6.7) and (6.8) we conclude that $g=\Lambda h_{\chi}$ satisfies (6.4).

Using Kato's inequality it is straightforward to extend Theorem 6.1 to the case of real magnetic fields:

Theorem 6.2. Suppose that $V$ satisfies hypothesis (i) and (ii) of Theorem 6.1. Let a $(x)$ be a real $C^{1}$-vector field on $R^{v}$. Then $\pi_{k}=p_{k}+a_{k}(x)(k=1 \ldots v)$ and $A_{0}=\pi^{2}+V$ are defined on $C_{0}^{\infty}\left(R^{v}\right)$, and there exist constants $\xi, \eta$ depending only on $\alpha, \beta, \gamma$ such that

$$
\left(u, \pi^{2} u\right) \leqq \xi\left\{\cos \gamma \operatorname{Re}\left(u, A_{0} u\right)+\sin \gamma \operatorname{Im}\left(u, A_{0} u\right)+\eta(u, u)\right\}
$$

for all $u \in C_{0}^{\infty}\left(R^{v}\right)$. If $V_{1}$ also satisfies hypothesis (iii) of Theorem 6.1 then $\operatorname{Ran}\left(z+A_{0}\right)$ is dense for sufficiently large real $z$.

Proof. The proof of Theorem 6.1 extends immediately to this case due to the following result [3, Theorem 2.4]: if a multiplication operator has relative bound $<\alpha$ with respect to $p^{2}$ it also has relative bound $<\alpha$ with respect to $\pi^{2}$, uniformly in $a \in C^{1}$.

Analytic families. In the discussion of Examples 1 and 3 we have mentioned that analytic perturbation theory applies for $\kappa$ in the interior of the relevant sector. In this connection the following criterion is useful:

Lemma 6.3. Let $A(\kappa)$ be a family of closed operators on a Hilbert space $\mathscr{H}$, defined for $\kappa$ in some open complex set $G$. Suppose that $D$ is a common core of $A(\kappa)$ for all $\kappa \in G$. Then $A(\kappa)$ is an analytic family in the sense of Kato [8, Chap. VII] if there exists a sequence of analytic families $A_{n}(\kappa)$ on $G$ such that

(i) $\lim _{n \rightarrow \infty} A_{n}(\kappa) u=A(\kappa) u$ for all $u \in D$ and all $\kappa \in G$, and

(ii) for some $z \in C, R_{n}(z, \kappa)=\left(z-A_{n}(\kappa)\right)^{-1}$ and $R(z, \kappa)=(z-A(\kappa))^{-1}$ exist and are bounded uniformly in $n$ and $\kappa$.

Proof. Let $u, v \in \mathscr{H}$. By Lemma 1.2 the functions $f_{n}(\kappa)=\left(u, R_{n}(z, \kappa) v\right)$ converge to $f(\kappa)=(u, R(z, \kappa) v)$ for all $\kappa \in G$. Since they are analytic in $\kappa \in G$ and uniformly bounded, it follows from Vitali's theorem that $f(\kappa)$ is analytic in $G$. Since $u, v$ are arbitrary, $R(z, \kappa)$ is analytic in $\kappa \in G$.

Typically, $A_{n}(\kappa)$ is obtained by putting a cut-off on some singular part of $A(\kappa)$, e.g.

$$
A_{n}(\kappa)=p^{2}+x^{2}+\kappa x^{4} e^{-x^{2} / n}
$$

in the case of Example 1. Then (i) is obvious for $u \in C_{0}^{\infty}\left(R^{1}\right)$ and (ii) follows for real $z<0$ by inspecting the numerical ranges of $A_{n}(\kappa)$ and $A(\kappa)$. 


\section{References}

1. Aguilar, J., Combes, J.M.: Commun. Math. Phys. 22, 269-279 (1971)

2. Aventini, P., Seiler, R.: Commun. Math. Phys. 41, 119-134 (1975)

3. Avron, J.E., Herbst, I.W., Simon, B.: Duke Math. J. 45, 847-883 (1978)

4. Avron, J.E., Herbst, I.W., Simon, B.: Commun. Math. Phys. 79, 529-574 (1981)

5. Enss, V.: Commun. Math. Phys. 52, 233-238 (1977)

6. Herbst, I.W.: Commun. Math. Phys. 64, 279-298 (1979)

7. Herbst, I.W., Simon, B. : Commun. Math. Phys. 80, 181-216 (1981)

8. Kato, T.: Perturbation theory for linear operators. Berlin, Heidelberg, New York: Springer 1966

9. Morgan III, J.D., Simon, B.: Int. J. Quantum Chem. 17, 1143-1166 (1980)

10. Reed, M., Simon, B.: Methods of modern mathematical physics. II. Fourier analysis, selfadjointness. New York: Academic Press 1975

11. Reed, M., Simon, B.: Methods of modern mathematical physics. IV. Analysis of operators. New York: Academic Press 1978

12. Schechter, M. : Spectra of partial differential operators. Amsterdam, London: North-Holland 1971

13. Weyl, H.: Rend. Circ. Mat. Palermo 27, 373-392 (1909); Gesammelte Abhandlungen, Vol. I, pp. 175-194. Berlin, Heidelberg, New York: Springer 1968

Communicated by B. Simon

Received June 18, 1980; in revised form September 22, 1981 